Bull. Austral. Math. Soc.

VOL. 58 (1998) [177-187]

\title{
THE HEAT FLOWS AND HARMONIC MAPS FROM COMPLETE MANIFOLDS INTO REGULAR BALLS
}

\author{
Jiayu Li and SILEI WaNG
}

We generalise the existence result for harmonic maps obtained by Hildebrandt-Kaul-

Widman to the case where the domain manifold is complete noncompact.

\section{INTRODUCTION}

Let $M$ and $N$ be two Riemannian manifolds of dimension $m$ and $n$. Suppose their metrics are given by $d s_{M}^{2}=g_{i j} d x^{i} d x^{j}$ and $d s_{N}^{2}=h_{\alpha \beta} d u^{\alpha} d u^{\beta}$. The energy density function of $\mathrm{u}$ is given by

$$
e(u)=g^{i j} \frac{\partial u^{\alpha}}{\partial x^{i}} \frac{\partial u^{\beta}}{\partial x^{j}} h_{\alpha \beta}=|\nabla u|^{2} .
$$

The total energy is defined by

$$
E(u)=\int_{M} e(u) d x
$$

A mapping $u: M \longrightarrow N$ is called a harmonic map if it is a classical solution of the Euler-Lagrange equation of $E(u)$, which can be written as

$$
\tau^{\alpha}(u(x))=\Delta u^{\alpha}(x)+\Gamma_{\beta \gamma}^{\alpha}(u(x)) \frac{\partial u^{\beta}}{\partial x^{i}} \frac{\partial u^{\gamma}}{\partial x^{j}} g^{i j}=0
$$

where $\tau(u)$ is called the tension field of $\mathrm{u}$. The corresponding parabolic system with initial data $u_{0}(x)$, known as the the heat equation for harmonic maps, is as follows

$$
\left\{\begin{array}{l}
\frac{\partial u}{\partial t}=\tau(u) \\
u(x, 0)=u_{0}(x)
\end{array}\right.
$$

If $M$ is a compact Riemannian manifold with boundary $\partial M$, then we may consider the following Dirichlet problems:

$$
\left\{\begin{array}{l}
\tau(u)=0 \\
\left.u\right|_{\partial M}=h_{0}
\end{array}\right.
$$

Received 15th December, 1997

The first author would like to express his gratitude to Professor W.-Y. Ding for his long time encouragement. He would also like to thank Dr.Y, Wang for his helpful discussions.

Copyright Clearance Centre, Inc. Serial-fee code: 0004-9729/98 \$A2.00+0.00. 
and

$$
\left\{\begin{array}{l}
\frac{\partial u}{\partial t}=\tau(u) \\
u(x, 0)=u_{0}(x) \\
\left.u(\cdot, t)\right|_{\partial M}=h_{0}(\cdot)
\end{array}\right.
$$

where $u_{0} \in C^{1}(M, N), h_{0} \in C^{1}(\partial M, N),\left.u_{0}\right|_{\partial M}=h_{0}$.

When $M$ and $N$ are compact without boundary and $N$ has nonpositive sectional curvature, Eells-Sampson [10] proved that any $C^{1}$ map from $M$ into $N$ can be deformed to a harmonic map, by solving (1.2). The analogous version for compact manifolds with boundary was proved by Hamilton [12], who solved the equation (1.4).

Hildebrandt-Kaul-Widman [15] solved the equation (1.3) when $h_{0}(\partial M)$ is contained in a regular ball. Later Jost [16] reproved the result by the heat flow method. In [1] Avilés-Choi-Micallef considered the case where the domain manifold $M$ is complete and simply connected with sectional curvature $K_{M}$ satisfying $-b^{2} \leqslant K_{M} \leqslant-a^{2}<0$. They proved that the Dirichlet problem at infinity for harmonic maps from $M$ into a regular ball has a solution, by using the continuity method.

Ding-Lin [8] showed that if $M$ and $N$ are compact without boundary and the universal covering of $N$ admits a strictly convex function with quadratic growth then the result of Eells-Sampson holds.

When $M$ is a complete noncompact Riemannian manifold and $N$ is a complete Riemannian manifold with nonpositive sectional curvature, $\mathrm{Li}$-Tam [22] proved the long time existence result for the initial value problem of (1.2), provided the initial data $u_{0}$ has bounded energy density. If we impose some conditions on $M$ and $u_{0}$ we can have the convergence of the heat flow $u(x, t)$ to a harmonic map from $M$ into $N$ (see $[9,20,22,23])$, which generalises the result of Eells-Sampson to the noncompact case.

But, it is well-known that the conclusion of Eells-Sampson can not be generally achieved and one has to pose some assumptions on $N$ to draw such a conclusion because the solution of (1.2) and the solution of (1.4) may blow up in finite time (see $[5,6,7])$.

In this paper, we assume that $M$ is a complete noncompact Riemannian manifold.

To state our conclusions we introduce some definitions.

DEFinition 1.1: Let $N$ be a Riemannian manifold and $\Omega$ a bounded open subset of $N$. We say $\Omega$ satisfies condition (B) if there exists a positive function $f \in C^{2}(\Omega)$ satisfying

$$
\begin{gathered}
-\nabla^{2} f-f K_{2}(y) h \geqslant C_{0}(\Omega) h \\
0<m_{1}(\Omega) \leqslant f(y) \leqslant m_{2}(\Omega)<\infty
\end{gathered}
$$

and

$$
|\nabla f(y)| \leqslant m_{3}(\Omega)<\infty
$$


for all $y \in \Omega$, where $K_{2}(y)=\sup \{K(y, \pi), 0 \mid K(y, \pi)$ is the sectional curvature of a two plane $\left.\pi \in T_{y} N\right\}$, and $C_{0}(\Omega)>0$.

DEFINITION 1.2: If $\Omega$ satisfies condition (B) and there is a nonnegative convex function $f^{*}$ on $\Omega$ such that $\Omega=\left(f^{*}\right)^{-1}([0, r))$, we call $\Omega$ a generalised regular ball.

EXAMPLE 1.3. Suppose $N$ is a Riemannian manifold with nonpositive sectional curvature. If $\Omega \subset N$ is a open bounded subset and there is a point $y_{0} \in N$ such that $\Omega \cap C u t\left(y_{0}\right)=\emptyset$, then $\Omega$ satisfies condition (B). $B_{r}\left(y_{0}\right)$ is a generalised regular ball if $B_{r}\left(y_{0}\right) \cap C u t\left(y_{0}\right)=\emptyset$.

EXAMPLE 1.4. Suppose $N$ is a Riemannian manifold, $B_{r}\left(y_{0}\right)$ is a regular ball (see [13]), that is $C u t\left(y_{0}\right) \cap B_{r}\left(y_{0}\right)=\emptyset$, and $\sqrt{K} r<\pi / 2$ where $K \geqslant 0$ is an upper bound of the sectional curvature of $N$ on $B_{r}\left(y_{0}\right)$, then $B_{r}(y o)$ is a generalised regular ball.

In this paper we mainly consider the heat flows and harmonic maps from a complete noncompact Riemannian manifold into a regular ball.

For the heat flows we prove

THEOREM 1.5. Let $M$ be a complete noncompact Riemannian manifold. Suppose that $\Omega$ is a regular ball. Assume that $u_{0} \in C^{1}(M, \Omega)$. Then (1.2) has a smooth solution $u(x, t)$ satisfying $u\left(B_{R}\left(x_{0}\right) \times[0, T)\right) \subset \subset \Omega$ for all $R>0$ and $T>0$.

Such a solution is unique if $M$ is compact or if there exists a point $x_{1} \in M$ and a positive constant $\beta$ such that $\operatorname{Vol}\left(B_{R}\left(x_{1}\right)\right) \leqslant \exp \left(\beta\left(1+R^{2}\right)\right)$ for all $R>0$.

For the convergence of the heat flow, we prove

THEOREM 1.6. Let $M$ be a complete noncompact Riemannian manifold. Let $\Omega$ be a regular ball. Assume that $u_{0} \in C^{1}(M, \Omega)$, and $E\left(u_{0}\right)<\infty$. Then (1.2) has a smooth solution $u(x, t)$ satisfying that $u\left(B_{R}\left(x_{0}\right) \times[0, T)\right) \subset \subset \Omega$ for all $R>0$ and $T>0$ and that there exists a subsequence $t_{v} \longrightarrow \infty$ such that $u\left(x, t_{v}\right) \longrightarrow u_{\infty}(x)$, and $u_{\infty}(x)$ is a harmonic map.

REMARK 1.7. If $M$ is a complete noncompact Riemannian manifold which admits a compact convex exhaustion $\left\{\Omega_{i}\right\}\left(\partial \Omega_{i}\right.$ is convex). Then by applying the existence theorem in [21] instead of using Jost's result in the proof of Theorem 3.1, we can prove the condition in Theorem 1.5 and Theorem 1.6 that $\Omega$ is a regular ball can be replaced by the assumption that $\Omega$ is a generalised regular ball.

The main idea in the proof of our theorems is using the gradient estimates for the heat flow of harmonic maps derived in section 2 which can be seen as the parabolic version of Cheng's result [3] and Jost's result [17]. The estimates are of interest in their own right. And as a consequence we can derive a Liouville theorem for harmonic maps which is similar to a result of Hildebrandt-Jost-Widman [14] and generalise the results of Cheng [3], Choi [4] and Yu [25] (also see [18]).

In this paper $C_{m}$ denotes various constants which depends only on m; similarly $C$ denotes various universal constants. 


\section{Gradient Estimates}

In this section we derive some gradient estimates for the heat flow of harmonic maps, which are essential in the proof of our main results. At the end of this section, we shall prove a Liouville theorem for harmonic maps.

THEOREM 2.1. Let $M$ and $N$ be Riemannian manifolds. Let $x_{0} \in M$ and $r(x)$ be the distance function from $x_{0}$, and let $B_{R}\left(x_{0}\right)=\{x \in M \mid r(x) \leqslant R\}$. Suppose that $\Omega \subset N$ satisfies condition (B) and assume that the Ricci curvature of $M$ on $B_{R}\left(x_{0}\right)$ is bounded from below by $-K_{1} \leqslant 0$. If $u(x, t)$ is a solution of the equation (1.2) on $B_{R}\left(x_{0}\right) \times\left[0, T_{1}\right), u\left(B_{R}\left(x_{0}\right) \times\left[0, T_{1}\right)\right) \subset \Omega$ and $B_{R}\left(x_{0}\right) \cap \partial M=\emptyset$ then

$$
\sup _{B_{R / 2}\left(x_{0}\right)}|\nabla u(x, t)| \leqslant C_{m} \frac{m_{2}}{\sqrt{C_{0} m_{1}}}\left(\sqrt{K_{1}}+\sqrt{\frac{K_{1}}{R}}+\frac{1}{R}\right)+C \frac{m_{2} m_{3}}{m_{1} C_{0}} \frac{1}{R}+\frac{m_{2}}{\sqrt{2 C_{0} m_{1}}} \frac{1}{\sqrt{t}}
$$

and

$$
\sup _{B_{R / 2}\left(x_{0}\right)}|\nabla u(x, t)| \leqslant \frac{m_{2}}{m_{1}} \sup _{B_{R}\left(x_{0}\right)}|\nabla u(x, 0)|+C_{m} \frac{m_{2}}{\sqrt{C_{0} m_{1}}}\left(\sqrt{K_{1}}+\sqrt{\frac{K_{1}}{R}}+\frac{1}{R}\right)+C \frac{m_{2} m_{3}}{C_{0} m_{1}} \frac{1}{R}
$$

for all $0<t<T_{1}$.

To prove the theorem we introduce

$$
\varphi_{0}(x, t)=\frac{|\nabla u(x, t)|^{2}}{f^{2}(u(x, t))}
$$

and estimate $\left(\Delta-\frac{\partial}{\partial t}\right) \varphi_{0}$. A straightforward computation gives

$$
\begin{gathered}
\nabla \varphi_{0}=\frac{\nabla|\nabla u|^{2}}{f^{2}}-2 \frac{\nabla f|\nabla u|^{2}}{f^{3}} \\
\Delta \varphi_{0}=\frac{\Delta|\nabla u|^{2}}{f^{2}}-4 \frac{\nabla f \nabla|\nabla u|^{2}}{f^{3}}-2 \frac{\Delta f|\nabla u|^{2}}{f^{3}}+6 \frac{|\nabla f|^{2}|\nabla u|^{2}}{f^{4}}
\end{gathered}
$$

and

$$
\frac{\partial \varphi_{0}}{\partial t}=\frac{\frac{\partial}{\partial t}|\nabla u|^{2}}{f^{2}}-2 \frac{\frac{\partial f}{\partial t}|\nabla u|^{2}}{f^{3}}
$$

We therefore have

$$
\begin{array}{r}
\left(\Delta-\frac{\partial}{\partial t}\right) \varphi_{0}=\frac{\left(\Delta-\frac{\partial}{\partial t}\right)|\nabla u|^{2}}{f^{2}}-2 \frac{\left(\Delta-\frac{\partial}{\partial t}\right) f(u(x, t))|\nabla u|^{2}}{f^{3}} \\
-4 \frac{\nabla f \nabla|\nabla u|^{2}}{f^{3}}+6 \frac{|\nabla f|^{2}|\nabla u|^{2}}{f^{4}}
\end{array}
$$


Using the Weitzenböck formula we have

$$
\begin{aligned}
\left(\triangle-\frac{\partial}{\partial t}\right)|\nabla u(x, t)|^{2}= & 2|\nabla d u|^{2}-2 \sum_{i, j}\left\langle R^{N}\left(d u\left(e_{i}\right), d u\left(e_{j}\right)\right) d u\left(e_{i}\right), d u\left(e_{j}\right)\right\rangle \\
& +2 \sum_{i}\left\langle d u\left(\operatorname{Ric}^{M}\left(e_{i}\right)\right), d u\left(e_{i}\right)\right\rangle
\end{aligned}
$$

where $e_{1}, e_{2}, \cdots, e_{n}$ is a local orthonormal frame field. Computing directly [3], one has

$$
\left(\triangle-\frac{\partial}{\partial t}\right) f(u(x, t))=\nabla^{2}(f)(\nabla u, \nabla u) .
$$

Since $\Omega$ satisfies condition (B), by substituting (2.3) and the last two identities into (2.4) we have

$$
\left(\Delta-\frac{\partial}{\partial t}\right) \varphi_{0} \geqslant-2 K_{1} \frac{|\nabla u|^{2}}{f^{2}}+2 C_{0} \frac{|\nabla u|^{4}}{f^{3}}+2 \frac{|\nabla d u|^{2}}{f^{2}}
$$

$$
-2 \frac{\nabla f \cdot \nabla|\nabla u|^{2}}{f^{3}}+2 \frac{|\nabla f|^{2}|\nabla u|^{2}}{f^{4}}-2 \nabla \varphi_{0} \cdot \frac{\nabla f}{f}
$$

The Hölder's inequality implies

$$
2 \frac{|\nabla d u|^{2}}{f^{2}}+2 \frac{|\nabla f|^{2}|\nabla u|^{2}}{f^{4}} \geqslant 4 \frac{|\nabla d u||\nabla u||\nabla f|}{f^{3}}
$$

and

$$
\left.|\nabla| \nabla u\right|^{2}|\leqslant 2| \nabla d u|| \nabla u \mid
$$

Substituting the last two inequalities into (2.5), we have

$$
\left(\Delta-\frac{\partial}{\partial t}\right) \varphi_{0} \geqslant 2 C_{0} m_{1} \varphi_{0}^{2}-2 \nabla \varphi_{0} \cdot \frac{\nabla f}{f}-2 K_{1} \varphi_{0} .
$$

Now we prove Theorem 2.1. For this purpose we introduce $F(x, t)=t \varphi_{0}(x, t)$. We obviously have from (2.6)

$$
\left(\Delta-\frac{\partial}{\partial t}\right) F \geqslant 2 m_{1} C_{0} \frac{F^{2}}{t}-\left(2 K_{1}+\frac{1}{t}\right) F-2 \nabla F \cdot \frac{\nabla f}{f} .
$$

Let $\psi(r)$ be a $C^{2}$ function on $[0, \infty)$ such that

$$
\psi(r)= \begin{cases}1 & \text { if } r \in[0,1 / 2] \\ 0 & \text { if } r \in[1, \infty)\end{cases}
$$

$0 \leqslant \psi(r) \leqslant 1, \psi^{\prime}(r) \leqslant 0, \psi^{\prime \prime}(r) \geqslant-C$ and $\left|\psi^{\prime}(r)\right|^{2} /(\psi(r)) \leqslant C$ where $C$ is an absolute constant. Let $g(x)=\psi(r(x) / R)$. 
Assume that $\left(x_{1}, t_{1}\right)$ is the point where $g F$ achieves its maximum in $B_{R}\left(x_{0}\right) \times$ $[0, T]\left(0<T<T_{1}\right)$. By using the argument of Calabi [2], we may assume $g(x)$ to be smooth at $x_{1}$. And we may also assume $(g F)\left(x_{1}, t_{1}\right)>0$. By the maximum principle, at $\left(x_{1}, t_{1}\right)$ we have

$$
\begin{aligned}
& \nabla(g F)=0, \\
& \frac{\partial}{\partial t}(g F) \geqslant 0,
\end{aligned}
$$

and

$$
\triangle(g F) \leqslant 0
$$

Hence

$$
\left(\Delta-\frac{\partial}{\partial t}\right) g F \leqslant 0
$$

Applying the Laplacian comparison theorem [11] we have

$$
r \Delta r \leqslant C_{m}\left(1+K_{1} r\right)
$$

So,

$$
\frac{|\nabla g|^{2}}{g} \leqslant \frac{C}{R^{2}}, \quad \triangle g \geqslant-\frac{C_{m}}{R^{2}}-\frac{C_{m} K_{1}}{R} .
$$

By (2.9) and the last two inequalities we have

$$
0 \geqslant-\left(\frac{C_{m}}{R^{2}}+\frac{C_{m} K_{1}}{R}\right) F+2 \nabla g \nabla F+g\left(\Delta-\frac{\partial}{\partial t}\right) F
$$

Substituting (2.7) and (2.8) into (2.10) we have

$$
\begin{aligned}
0 \geqslant-\left(\frac{C_{m}}{R^{2}}\right. & \left.+\frac{C_{m} K_{1}}{R}\right) F-2 \frac{|\nabla g|^{2}}{g} F+g\left(\triangle-\frac{\partial}{\partial t}\right) F \\
\geqslant & -\left(\frac{C_{m}}{R^{2}}+\frac{C_{m} K_{1}}{R}\right) F+2 m_{1} C_{0} \frac{1}{t_{1}} g F^{2} \\
& -\left(2 K_{1}+\frac{1}{t_{1}}\right) g F-m_{3} \frac{|\nabla g|}{g^{1 / 2} t_{1}^{1 / 2}}(g F)^{1 / 2} F
\end{aligned}
$$

where we have used $|\nabla f(u(x, t))| \leqslant m_{3}|\nabla u|$. Multiplying through (2.11) by $g$ and using $0 \leqslant g \leqslant 1$, we have

$$
0 \geqslant 2 m_{1} C_{0} \frac{1}{t_{1}}(g F)^{2}-\left(\frac{C_{m}}{R^{2}}+\frac{C_{m} K_{1}}{R}+2 K_{1}+\frac{1}{t_{1}}\right) g F-C m_{3} \frac{1}{R}\left(\frac{g F}{t_{1}}\right)^{1 / 2} g F .
$$


Using the quadratic formula one obtains

$$
\begin{aligned}
\left(\frac{g F}{t_{1}}\right)^{1 / 2} & \leqslant \frac{C m_{3}}{2 m_{1} C_{0} R}+\sqrt{\frac{1}{2 m_{1} C_{0}}\left(2 K_{1}+\frac{1}{t_{1}}+\frac{C_{m}}{R^{2}}+\frac{C_{m} K_{1}}{R}\right)} \\
& \leqslant C \frac{m_{3}}{m_{1} C_{0} R}+C_{m} \frac{1}{\sqrt{m_{1} C_{0}}}\left(\sqrt{K_{1}}+\sqrt{\frac{K_{1}}{R}}+\frac{1}{R}\right)+\frac{1}{\sqrt{t_{1}}} \frac{1}{\sqrt{2 C_{0} m_{1}}}, \\
(g F)^{1 / 2}\left(x_{1}, t_{1}\right) & \leqslant C_{m} \frac{1}{\sqrt{m_{1} C_{0}}}\left(\sqrt{K_{1}}+\sqrt{\frac{K_{1}}{R}}+\frac{1}{R}\right) \sqrt{T} C \frac{m_{3}}{m_{1} C_{0}} \frac{\sqrt{T}}{R}+\frac{1}{\sqrt{2 m_{1} C_{0}}} .
\end{aligned}
$$

So

$$
\begin{aligned}
& \sup \left\{t^{1 / 2}|\nabla u(x, t)| \mid(x, t) \in B_{R / 2}\left(x_{0}\right) \times[0, T]\right\} \\
& \leqslant C_{m} \frac{m_{2}}{\sqrt{m_{1} C_{0}}}\left(\sqrt{K_{1}}+\sqrt{\frac{K_{1}}{R}}+\frac{1}{R}\right) \sqrt{T}+C \frac{m_{2} m_{3}}{m_{1} C_{0}} \frac{\sqrt{T}}{R}+\frac{m_{2}}{\sqrt{2 m_{1} C_{0}}}
\end{aligned}
$$

and so

$$
\sup _{B_{R / 2}\left(x_{0}\right)} T^{1 / 2}|\nabla u(x, T)| \leqslant C_{m} \frac{m_{2} \sqrt{T}}{\sqrt{m_{1} C_{0}}}\left(\sqrt{K_{1}}+\sqrt{\frac{K_{1}}{R}}+\frac{1}{R}\right)+C \frac{m_{2} m_{3}}{m_{1} C_{0}} \frac{\sqrt{T}}{R}+\frac{m_{2}}{\sqrt{2 m_{1} C_{0}}},
$$

(2.1) follows.

To prove (2.2) we set $F(x, t)=\varphi_{0}(x, t)$. If $g F$ achieves its maximum in $B_{R / 2}\left(x_{0}\right) \times$ $[0, T]$ for $0<T<T_{1}$ at $\left(x_{1}, 0\right)$, then we have

$$
\sup _{B_{R / 2}\left(x_{0}\right)}|\nabla u(x, t)| \leqslant \frac{m_{2}}{m_{1}} \sup _{B_{R}\left(x_{0}\right)}|\nabla u(x, 0)| .
$$

If $g F$ achieves its maximum at $\left(x_{1}, t_{1}\right)\left(t_{1}>0\right)$, then by an argument similar to the one used in the proof of (2.1) we have

$$
\sup _{B_{R / 2}\left(x_{0}\right)}|\nabla u(x, t)| \leqslant C_{m} \frac{m_{2}}{\sqrt{m_{1} C_{0}}}\left(\sqrt{K_{1}}+\sqrt{\frac{K_{1}}{R}}+\frac{1}{R}\right)+C \frac{m_{2} m_{3}}{m_{1} C_{0}} \frac{1}{R}
$$

(2.2) follows from (2.12) and (2.13).

If instead of considering the function of $g F$ we consider $F$ directly, we have the following theorems.

THEOREM 2.2. Let $M$ be a compact Riemannian manifold, let $N$ be a Riemannian manifold, and let $\Omega \subset N$ satisfy condition (B). If $u(x, t)$ is a solution of the equation (1.2) on $M \times\left[0, T_{1}\right)$ and $u\left(M \times\left[0, T_{1}\right)\right) \subset \Omega$ then

$$
|\nabla u(x, t)| \leqslant C_{m} \frac{m_{2} \sqrt{K_{1}}}{\sqrt{m_{1} C_{0}}}+\frac{m_{2}}{\sqrt{2 C_{0} m_{1}}} \frac{1}{\sqrt{t}}
$$


and

$$
|\nabla u(x, t)| \leqslant \frac{m_{2}}{m_{1}} \sup _{M}|\nabla u(x, 0)|+C_{m} \frac{m_{2} \sqrt{K_{1}}}{\sqrt{C_{0} m_{1}}}
$$

for all $(x, t) \in M \times\left(0, T_{1}\right)$, where $-K_{1} \leqslant 0$ is the lower bound of the Ricci curvature of $M$.

For harmonic maps, as corollaries of Theorem 2.1 and Theorem 2.2 we have the following gradient estimates.

Theorem 2.3. Suppose that $M, N, B_{R}\left(x_{0}\right), \Omega$ satisfy the hypotheses of Theorem 2.1. If $u(x)$ is a harmonic map from $B_{R}\left(x_{0}\right)$ into $\Omega$ then

$$
\sup _{B_{R / 2}\left(x_{0}\right)}|\nabla u(x)| \leqslant C_{m} \frac{m_{2}}{\sqrt{C_{0} m_{1}}}\left(\sqrt{K_{1}}+\sqrt{\frac{K_{1}}{R}}+\frac{1}{R}\right)+C \frac{m_{2} m_{3}}{m_{1} C_{0}} \frac{1}{R} .
$$

Theorem 2.4. Suppose that $M, N, \Omega$ satisfy the hypotheses of Theorem 2.2 . If $u(x)$ is a harmonic map from $M$ into $\Omega$ then

$$
\sup _{M}|\nabla u(x)| \leqslant C_{m} \frac{m_{2} \sqrt{K_{1}}}{\sqrt{C_{0} m_{1}}} .
$$

By applying Theorem 2.3 we can obtain a Liouville theorem for harmonic maps.

THEOREM 2.5. Let $M$ be a complete Riemannian manifold with nonnegative Ricci curvature, and let $N$ be a Riemannian manifold. Suppose that $\Omega \subset N$ satisfies condition (B). If $u(x)$ is a harmonic map from $M$ into $\Omega$, then $u$ is constant.

\section{Heat Flows}

In this section we consider the global existence of the heat flow of harmonic maps from complete noncompact manifolds into regular balls.

ThEOREM 3.1. Let $M$ be a complete noncompact Riemannian manifold. Suppose that $\Omega$ is a regular ball. Assume $u_{0} \in C^{1}(M, \Omega)$. Then (1.2) has a smooth solution $u$ defined on $M \times[0, \infty)$ satisfying the properties

(a) $u\left(B_{R}\left(x_{0}\right) \times[0, T)\right) \subset \subset \Omega$

(b) $\sup _{B_{R}\left(x_{0}\right) \times[0, T)}|\nabla u|<\infty$ for all $R>0$ and $T>0$.

Such a solution is unique if there exists a point $x_{1} \in M$ and a positive constant $\beta$ such that $\operatorname{Vol}\left(B_{R}\left(x_{0}\right)\right) \leqslant \exp \left(\beta\left(1+R^{2}\right)\right)$ for all $R>0$.

PROOF: Choose a sequence of compact smooth domains $\Omega_{i}$ such that $\Omega_{i} \subset \Omega_{i+1}$ for all $i=1,2 \ldots$ and $B_{i}\left(x_{0}\right) \subset \Omega_{i}$ for some fixed point $x_{0} \in M$. By Jost's result [16] we can get a solution $u_{i}(x, t)$ of the following equation for each $i$.

$$
\left\{\begin{array}{l}
\frac{\partial u_{i}}{\partial t}=\tau\left(u_{i}\right) \\
u_{i}(x, 0)=u_{0}(x) \\
u_{i}(\cdot, t)=\left.u_{0}(\cdot)\right|_{\partial \Omega_{i}} \text { for all } t>0
\end{array}\right.
$$


satisfying $u_{i}\left(\Omega_{i} \times[0, T)\right) \subset \subset \Omega$ and $\sup _{\Omega_{i} \times[0, T)}\left|\nabla\left(u_{i}\right)\right|<\infty$ for all $i$ and $T>0$.

For any $R>0$, there exists an integer $i(R)>0$ such that $B_{2 R}\left(x_{0}\right) \subset \Omega_{i}$ for all $i \geqslant i(R)$. Theorem 2.1 yields

$$
\sup _{B_{R}\left(x_{0}\right) \times[0, T]}\left|\nabla u_{i}(x, t)\right| \leqslant \frac{m_{2}}{m_{1}} \sup _{B_{2 R}\left(x_{0}\right)}\left|\nabla u_{0}\right|+C_{m} \frac{m_{2}}{\sqrt{C_{0} m_{1}}}\left(\sqrt{K_{1}}+\sqrt{\frac{K_{1}}{R}}+\frac{1}{R}\right)+C \frac{m_{2} m_{3}}{C_{0} m_{1}} \frac{1}{R}
$$

for all $i \geqslant i(R)$ and $T>0$, where $-K_{1} \leqslant 0$ is the lower bound of the Ricci curvature of $M$ on $B_{2 R}\left(x_{0}\right)$.

By standard interior estimates for the equation (1.2) and the diagonal subsequence argument, there exists a subsequence $u_{j}$ and $u \in C^{2,1}(M \times[0, \infty), \Omega)$ such that $u_{j} \longrightarrow u$ in $C^{2,1}\left(B_{R}\left(x_{0}\right) \times[0, R], \Omega\right)$ for any $R>0$. Clearly $u(x, t)$ is a solution of (1.2) satisfying (a) and (b), and $\lim _{t \rightarrow 0} u(x, t)=u_{0}(x)$ uniformly in $B_{R}\left(x_{0}\right)$ for any $R>0$.

If $v$ is another solution of (1.2) satisfying (a), then we consider the distance function $d(u, v)$. We set $\phi(x, t)=d^{2}(u, v)$. Clearly [24] $\phi(x, t)$ is smooth on $M \times[0, \infty)$ and satisfies $\left(\triangle-\frac{\partial}{\partial t}\right) \phi \geqslant-K_{2} \phi$ where $K_{2}=\sup _{y \in \Omega} K_{2}(y)$. By the maximum principle [19] we know that $\phi(x, t) \leqslant e^{K_{2} t} \phi(x, 0)=0$ if there exists a point $x_{1} \in M$ and a positive constant $\beta$ such that $\operatorname{Vol}\left(B_{R}\left(x_{1}\right)\right) \leqslant \exp \left(\beta\left(1+R^{2}\right)\right)$ for all $R>0$. This establishes the theorem.

\section{HARMONIC MAPS}

In this section we consider the convergence of the heat flow to a harmonic map from a complete noncompact Riemannian manifold to a generalised regular ball.

ThEOREM 4.1. Let $M$ be a complete Riemannian manifold. Let $\Omega$ be a generalised regular ball. Suppose that $u_{0} \in C^{\mathbf{1}}(M, \Omega)$ and $E\left(u_{0}\right)<\infty$. If $u(x, t)$ is a solution of the equation (1.2) satisfying $u\left(B_{R}\left(x_{0}\right) \times[0, T)\right) \subset \Omega$ for all $R>0$ and $T>0$, then there exists a subsequence $t_{v} \longrightarrow \infty$ such that $u\left(x, t_{v}\right) \longrightarrow u_{\infty}(x)$ in $C^{2}\left(B_{R}\left(x_{0}\right), \Omega\right)$ for any $x_{0} \in M, R>0$, and $u_{\infty}(x)$ is a harmonic map.

Proof: It is clear that $u(x, t)$ satisfies

$$
E(u(\cdot, t))+2 \int_{0}^{t} \int_{M}\left|u_{t}\right|^{2} d x d t \leqslant E\left(u_{0}\right)
$$

which yields that

$$
\int_{0}^{\infty} \int_{M} u_{t}^{2} d x d t \leqslant E\left(u_{0}\right)
$$

Therefore there exists a subsequence $t_{v} \rightarrow \infty$ such that

$$
u_{t}\left(x, t_{v}\right) \longrightarrow 0
$$


weakly in $L^{2}(M)$. Theorem 2.1 yields

$$
\sup _{\boldsymbol{B}_{\boldsymbol{R}}\left(x_{0}\right) \times[0, \infty)}|\nabla u| \leqslant C\left(m, R, M, \Omega, u_{0}\right)
$$

By (4.2), and standard interior estimates for the equation (1.1) we may assume

$$
u\left(x, t_{v}\right) \longrightarrow u_{\infty}
$$

in $C^{2}\left(B_{R}\left(x_{0}\right), \Omega\right)$ for any $x_{0} \in M, R>0$.

(4.1) and (4.3) imply that $u_{\infty}$ is a weak harmonic map. Since $u_{\infty} \in C^{2}(M, \Omega), u_{\infty}$ is a harmonic map.

\section{REFERENCES}

[1] P. Avilés, H. Choi and M. Micallef, 'Boundary behavior of harmonic maps on non-smooth domains and complete negatively curved manifolds', J. Funct. Anal. 99 (1991), 293-311.

[2] E. Calabi, 'An extension of E. Hopf's maximum principle with an application to Riemannian geometry', Duke Math. J. 25 (1958), 45-46.

[3] S.Y. Cheng, 'Liouville theorem for harmonic maps', Proc. Sympos. Pure Math. 36 (1980), 147-151.

[4] H.I. Choi, 'On the Liouville theorem for harmonic maps', Proc. Amer. Math. Soc. 85 (1982), 91-94.

[5] Y. Chen and W.-Y. Ding, 'Blow-up and global existence for heat flows of harmonic maps', Invent. Math. (1990), 567-578.

[6] J.-M. Coron and J.-M. Ghidaglia, 'Explosion en temps fini pour le flot des applications harmoniques', C.R. Acad. Sci. Paris Sér. I Math. (1989), 339-344.

[7] W.-Y. Ding, 'Blow-up of solutions of heat flow for harmonic maps', Adv. in Math. 19 (1990), 80-92.

[8] W.-Y. Ding and F.-H. Lin, 'A generalization of Eells-Sampson's theorem', J. Partial Differential Equations 5 (1992), 13-22.

[9] W.-Y. Ding and Y. Wang, 'Harmonic maps of complete noncompact Riemannian manifolds', Internat. J. Math. 2 (1991), 617-633.

[10] J. Eells and J.H. Sampson, 'Harmonic mappings of Riemannian manifolds', Amer. J. Math. 86 (1964), 109-169.

[11] R.E. Greene and H. Wu, Function theory on manifolds which possess a pole, Lecture Notes in Math. 699 (Springer-Verlag, Berlin, Heidelberg, New York, 1979).

[12] R. Hamilton, Harmonic maps of manifolds with boundary, Lecture Notes in Math. 471 (Springer-Verlag, Berlin, Heidelberg, New York, 1974).

[13] S. Hildebrandt, 'Harmonic mappings of Riemannian manifolds', in Harmonic Maps and Minimal Immersions (Montecatini 1984), Lecture Notes in Math. 1161 (Springer-Verlag, Berlin, Heidelberg, New York, 1985).

[14] S. Hildebrandt, J. Jost and K-O. Widman, 'Harmonic mappings and minimal submanifolds', Invent. Math. 62 (1980), 269-298. 
[15] S. Hildebrandt, H. Kaul and K-O. Widman, 'An existence theorem for harmonic mappings of Riemannian manifolds', Acta Math. 138 (1977), 1-16.

[16] J. Jost, 'Ein Existenzbeweis für harmonische Abbildungen, die ein Dirichletproblem lösen, mittels der Methode des Wärmeflusses', Manuscripta Math. 34 (1981), 17-25.

[17] J. Jost, Harmonic mappings between Riemannian manifolds, Proc. Centre Math. Analysis 4 (Aust. Nat. Univ. Press, Canberra, Australia, 1983).

[18] W.S. Kendall, 'Probability, convexity, and harmonic maps with small image I: uniqueness and fine existence', Proc. London Math. Soc. 61 (1990), 371-406.

[19] K. Karp and P. Li, 'The heat equation on complete Riemannian manifolds', (unpublished).

[20] J. Li, 'The heat flows and harmonic maps of complete noncompact Riemannian manifolds', Math. Z. 212 (1993), 161-173.

[21] J. Li, 'Heat flows and harmonic maps with a free boundary', Math. Z. 217 (1994), 487-495.

[22] P. Li and L.-F. Tam, 'The heat equation and harmonic maps of complete manifolds', Invent. Math. 105 (1991), 1-46.

[23] G.G. Liao and L.-T. Tam, 'On the heat equation for harmonic maps from noncompact manifolds', Pacific J. Math. 153 (1992), 129-145.

[24] R. Schoen and S.-T. Yau, 'Compact group actions and the topology of manifolds with nonpositive curvature', Topology 18 (1979), 361-380.

[25] Q.-H. Yu, 'Bounded harmonic maps', Acta Math. Sinica 1 (1985), 16-21.

Institute of Mathematics

Academia Sinica

Beijing 100080

Peoples Republic of China
Department of Mathematics

Hangzhou University

Hangzhou 310028

Peoples Republic of China 